\title{
Data of Statistical Analysis
}

Statistical Analysis of Fig. 3

\begin{tabular}{|c|c|c|c|}
\hline \multirow[t]{2}{*}{ Conditions } & \multicolumn{3}{|c|}{ Sample Group $($ mean $\pm S D, n=6)$} \\
\hline & Cont. (-LPS) & $\begin{array}{c}0 \mu \mathrm{g} / \mathrm{mL} \text { CHOS } \\
(+\mathrm{LPS})\end{array}$ & $\begin{array}{c}100 \mu \mathrm{g} / \mathrm{mL} \text { CHOS } \\
(+\mathrm{LPS})\end{array}$ \\
\hline PMA & $2.93( \pm 3.21)$ & $123.59( \pm 5.79)$ & $97.50( \pm 5.40)$ \\
\hline p-value & & $<0.001^{a}$ & $<0.001^{a},<0.001^{a^{*}}$ \\
\hline VitD3 & $0.27( \pm 0.60)$ & $237.582( \pm 9.27)$ & $41.74( \pm 4.90)$ \\
\hline p-value & & $<0.001^{a}$ & $<0.001^{a},<0.001^{a^{*}}$ \\
\hline
\end{tabular}

$a$ and $a^{*}$ indicated significant; $a^{*}:$ versus control (-LPS); a: versus $0 \mu \mathrm{g} / \mathrm{mL}$ CHOS, $p<0.001$

\section{Statistical Analysis of Fig. 4}

\begin{tabular}{|c|c|c|c|c|}
\hline Conditions & \multicolumn{4}{|c|}{ Sample Group (medians; $25 \%, 75 \% ; n=6$ ) } \\
\hline & $\mathbf{0 ~ h}$ & $48 \mathrm{~h}$ & $72 \mathrm{~h}$ & $96 \mathrm{~h}$ \\
\hline$\%$ CD14 of PMA & $0.84(0,2.11)$ & $10.68(9.48,12.16)$ & $18.03(17.36,19.20)$ & $16.56(15.6,17.88)$ \\
\hline p-value & & 0.424 & $<0.001^{a}$ & $0.004^{b}$ \\
\hline$\%$ PI of PMA & $1.50(0.89,1.742)$ & $\begin{array}{c}23.85(23.37 \\
25.88)\end{array}$ & $10.51(9.93,11.06)$ & $23.87(23.25,25.52)$ \\
\hline p-value & & $<0.001^{a}$ & 0.425 & $<0.001^{a}$ \\
\hline$\%$ CD14 of VitD3 & $0.74(0,1.85)$ & $\begin{array}{c}80.74(77.22 \\
81.39)\end{array}$ & $0.15(0,0.35)$ & $95.16(94.01,95.85)$ \\
\hline$p$-value & & 0.424 & $<0.001^{a}$ & $<0.001^{a}$ \\
\hline$\%$ PI of VitD3 & $0.68(0.13,1.11)$ & $0.13(0,0.30)$ & $0.15(0,0.35)$ & $0(0.21,0.53)$ \\
\hline p-value & & 0.071 & 0.146 & 0.427 \\
\hline
\end{tabular}

$p$-value versus control $(0 \mathrm{~h}) ; a=$ sig. $<0.001 ; b=$ sig. $<0.01$ 
Statistical Analysis of Fig. 5

\begin{tabular}{|c|c|c|c|c|c|c|c|c|}
\hline \multirow[b]{2}{*}{ Conditions } & \multicolumn{8}{|c|}{ Sample Groups (medians; $25 \%, 75 \% ; n=9$ ) } \\
\hline & $0 \mu \mathrm{g} / \mathrm{mL}$ & $\begin{array}{c}5 \mu \mathrm{g} / \mathrm{mL} \\
\mathrm{CHOS}\end{array}$ & $\begin{array}{c}10 \mu \mathrm{g} / \mathrm{mL} \\
\text { CHOS }\end{array}$ & $\begin{array}{c}25 \mu \mathrm{g} / \mathrm{mL} \\
\text { CHOS }\end{array}$ & $\begin{array}{c}50 \mu \mathrm{g} / \mathrm{mL} \\
\text { CHOS }\end{array}$ & $\begin{array}{c}100 \mu \mathrm{g} / \mathrm{mL} \\
\text { CHOS }\end{array}$ & $\begin{array}{c}200 \mu \mathrm{g} / \mathrm{mL} \\
\text { CHOS }\end{array}$ & $\begin{array}{c}100 \mathrm{ng} / \mathrm{mL} \\
\text { LPS }\end{array}$ \\
\hline$\%$ Cell viability & $\begin{array}{c}102.3 \\
(98.90,102.7)\end{array}$ & $\begin{array}{c}103.6 \\
(97.84,105.1)\end{array}$ & $\begin{array}{c}102.9 \\
(101.3,104.4)\end{array}$ & $\begin{array}{c}106.2 \\
(105.5,107.4)\end{array}$ & $\begin{array}{c}101.8 \\
(92.13,105.2)\end{array}$ & $\begin{array}{c}102.6 \\
(96.95,106.5)\end{array}$ & $\begin{array}{c}104.8 \\
(100.5,109.2)\end{array}$ & $\begin{array}{c}98.54 \\
(95.14,99.77)\end{array}$ \\
\hline$p$-value & & $>0.999$ & $>0.999$ & $>0.999$ & 0.123 & $>0.999$ & $>0.999$ & $>0.999$ \\
\hline
\end{tabular}

p-value versus control $(0 \mu \mathrm{g} / \mathrm{mL})$ 
Statistical Analysis of Fig. 6

\begin{tabular}{|c|c|c|c|c|c|c|c|c|}
\hline \multirow[b]{2}{*}{ Conditions } & \multicolumn{8}{|c|}{ Sample Groups (medians; $25 \%, 75 \% ; n=6$ ) } \\
\hline & Control & $0.5 \mu \mathrm{M}$ Daxa & $\begin{array}{c}5 \mu \mathrm{g} / \mathrm{mL} \\
\text { CHOS }\end{array}$ & $\begin{array}{c}10 \mu \mathrm{g} / \mathrm{mL} \\
\mathrm{CHOS}\end{array}$ & $\begin{array}{c}25 \mu \mathrm{g} / \mathrm{mL} \\
\text { CHOS }\end{array}$ & $\begin{array}{c}50 \mu \mathrm{g} / \mathrm{mL} \\
\text { CHOS }\end{array}$ & $\begin{array}{c}100 \mu \mathrm{g} / \mathrm{mL} \\
\text { CHOS }\end{array}$ & $\begin{array}{c}200 \mu \mathrm{g} / \mathrm{mL} \\
\text { CHOS }\end{array}$ \\
\hline $\begin{array}{l}\text { Non LPS-stimulated } \\
(\mathrm{IL}-1 \beta)\end{array}$ & 0.00 & 0.00 & 0.00 & $0(0,0.46)$ & $\begin{array}{c}2.32 \\
(0,5.56)\end{array}$ & $\begin{array}{c}8.11 \\
(0,20.44)\end{array}$ & $\begin{array}{c}21.46 \\
(5.42,36.08)\end{array}$ & $\begin{array}{c}36.57 \\
(19.08,54.71)\end{array}$ \\
\hline$p$-value & - & $>0.999$ & $>0.999$ & $>0.999$ & $>0.999$ & 0.575 & $0.005^{b}$ & $>0.001^{a}$ \\
\hline $\begin{array}{l}\text { Non LPS-stimulated } \\
\text { (IL-6) }\end{array}$ & 0.00 & 0.00 & 0.00 & 0.00 & 0.00 & $\begin{array}{c}0 \\
(0,0.5)\end{array}$ & $\begin{array}{c}3.45 \\
(0,7.58)\end{array}$ & $\begin{array}{c}9.22 \\
(0,19.79)\end{array}$ \\
\hline$p$-value & - & $>0.999$ & $>0.999$ & $>0.999$ & $>0.999$ & $>0.999$ & 0.176 & 0.081 \\
\hline $\begin{array}{l}\text { Non LPS-stimulated } \\
(\mathrm{TNF}-\alpha)\end{array}$ & 0.00 & 0.00 & 0.00 & 0.00 & 0.00 & $\begin{array}{c}1.59 \\
(0,5.67)\end{array}$ & $\begin{array}{c}7.83 \\
(0,18.98)\end{array}$ & $\begin{array}{c}19.45 \\
(0,40.32)\end{array}$ \\
\hline$p$-value & - & $>0.999$ & $>0.999$ & $>0.999$ & $>0.999$ & 0.395 & 0.205 & 0.099 \\
\hline $\begin{array}{l}\text { LPS-stimulated } \\
(\mathrm{IL}-1 \beta)\end{array}$ & $\begin{array}{c}301.2 \\
(274.4,334.6)\end{array}$ & $\begin{array}{c}62.57 \\
(54.8,69.28)\end{array}$ & $\begin{array}{c}274.8 \\
(264.6,295.8)\end{array}$ & $\begin{array}{c}237.2 \\
(231.7,245.9)\end{array}$ & $\begin{array}{c}135.5 \\
(105.2,164.4)\end{array}$ & $\begin{array}{c}90.75 \\
(79.28,107.0)\end{array}$ & $\begin{array}{c}65.74 \\
(63.73,68.37)\end{array}$ & $\begin{array}{c}67.46 \\
(58.02,80.44)\end{array}$ \\
\hline$p$-value & - & $<0.001^{a}$ & $>0.999$ & $>0.999$ & 0.236 & $0.038^{c}$ & $<0.001^{a}$ & $<0.001^{a}$ \\
\hline $\begin{array}{l}\text { LPS-stimulated } \\
\text { (IL-6) }\end{array}$ & $\begin{array}{c}266.3 \\
(262.3,270.2)\end{array}$ & $\begin{array}{c}2.25 \\
(0,4.67)\end{array}$ & $\begin{array}{c}211.2 \\
(191.3,190.6)\end{array}$ & $\begin{array}{c}173.6 \\
(145.5,190.6)\end{array}$ & $\begin{array}{c}112.3 \\
(102.1,123.4)\end{array}$ & $\begin{array}{c}77.72 \\
(74.35,81.3)\end{array}$ & $\begin{array}{c}53.79 \\
(47.35,62.12)\end{array}$ & $\begin{array}{c}50.15 \\
(42.15,61.83)\end{array}$ \\
\hline$p$-value & - & $<0.001^{a}$ & $>0.999$ & $>0.999$ & 0.182 & $0.021^{c}$ & $<0.001^{a}$ & $<0.001^{a}$ \\
\hline $\begin{array}{l}\text { LPS-stimulated } \\
(\mathrm{TNF}-\alpha) \\
\\
\quad \boldsymbol{p} \text {-value }\end{array}$ & $\begin{array}{c}797 \\
(758,827) \\
-\end{array}$ & $\begin{array}{c}223 \\
(190,261) \\
0.02^{c}\end{array}$ & $\begin{array}{c}674 \\
(618,678) \\
>0.999\end{array}$ & $\begin{array}{c}555 \\
(535,561) \\
0.963\end{array}$ & $\begin{array}{c}300 \\
(313,334) \\
0.182\end{array}$ & $\begin{array}{c}172 \\
(152,182) \\
0.002^{b}\end{array}$ & $\begin{array}{c}78.7 \\
(75.2,82.4) \\
<0.001^{a}\end{array}$ & $\begin{array}{c}65.1 \\
(49.7,78.5) \\
<0.001^{a}\end{array}$ \\
\hline $\begin{array}{l}\text { LPS-stimulated (n=3) } \\
\text { (IL-1b; } \\
\text { Washing CHOS) }\end{array}$ & $\begin{array}{c}45.62 \\
(42.76,51.06)\end{array}$ & - & - & - & - & $\begin{array}{c}21.24 \\
(17.22,34.19)\end{array}$ & $\begin{array}{c}12.76 \\
(10.44,16.24)\end{array}$ & - \\
\hline$p$-value & - & & & & & 0.359 & $0.015^{c}$ & \\
\hline
\end{tabular}

p-value versus control; $a=$ sig. $<0.001 ; b=$ sig. $<0.01 ; c=$ sig. $<0.05$ 
Statistical Analysis of Fig. 7

\begin{tabular}{|c|c|c|c|c|}
\hline \multirow{2}{*}{ Conditions } & \multicolumn{4}{|c|}{ Sample Groups (medians; 25\%, 75\%; $n=6$ ) } \\
\hline & Control & CHOS & VitD3 alone & VitD3 (CHOS) \\
\hline $\begin{array}{l}\text { Non LPS-stimulated } \\
(\% \text { CD } 14)\end{array}$ & $0.87(0.78,2.46)$ & $2.12(1.60,2.53)$ & $81.7(80.86 .3,83.35)$ & $90.46(90.17,95.1)$ \\
\hline$p$-value & & $>0.999$ & 0.054 & $<0.001^{a}, 0.85^{n s}$ \\
\hline $\begin{array}{l}\text { LPS-stimulated } \\
(\% \text { CD } 14)\end{array}$ & $1.37(0.78,1.48)$ & $2.41(1.38,2.56)$ & $91.37(88.14 .3,95.15)$ & $94.36(93.6,95.48)$ \\
\hline p-value & & $>0.999$ & $0.012^{c}$ & $0.001^{b},>0.999^{n s}$ \\
\hline
\end{tabular}

$p$-value versus control; $a=$ sig. $<0.001) ; b=$ sig. $<0.01 ; c=$ sig. $<0.05 ; n s=$ not significant when versus VitD3 alone 